\title{
Role of radiotherapy in the management of brain metastases of NSCLC - Decision criteria in clinical routine
}

Glatzer, Markus ; Faivre-Finn, Corinne ; De Ruysscher, Dirk ; Widder, Joachim ; Van Houtte, Paul ; Troost, Esther G C ; Slotman, Ben J ; Ramella, Sara ; Pöttgen, Christoph ; Peeters, Stephanie T H ; Nestle, Ursula ; McDonald, Fiona ; Le Pechoux, Cecile ; Dziadziuszko, Rafal ; Belderbos, José ; Ricardi, Umberto ; Manapov, Farkhad ; Lievens, Yolande ; Geets, Xavier ; Dieckmann, Karin ; Guckenberger, Matthias ; Andratschke, Nicolaus ; Süveg, Krisztian ; Putora, Paul M

\begin{abstract}
Background Whole brain radiotherapy (WBRT) is a common treatment option for brain metastases secondary to non-small cell lung cancer (NSCLC). Data from the QUARTZ trial suggest that WBRT can be omitted in selected patients and treated with optimal supportive care alone. Nevertheless, WBRT is still widely used to treat brain metastases secondary to NSCLC. We analysed decision criteria influencing the selection for WBRT among European radiation oncology experts. Methods Twenty-two European radiation oncology experts in lung cancer as selected by the European Society for Therapeutic Radiation Oncology (ESTRO) for previous projects and by the Advisory Committee on Radiation Oncology Practice (ACROP) for lung cancer were asked to describe their strategies in the management of brain metastases of NSCLC. Treatment strategies were subsequently converted into decision trees and analysed for agreement and discrepancies. Results Eight decision criteria (suitability for SRS, performance status, symptoms, eligibility for targeted therapy, extra-cranial tumour control, age, prognostic scores and "Zugzwang" (the compulsion to treat)) were identified. WBRT was recommended by a majority of the European experts for symptomatic patients not suitable for radiosurgery or fractionated stereotactic radiotherapy. There was also a tendency to use WBRT in the ALK/EGFR/ROS1 negative NSCLC setting. Conclusion Despite the results of the QUARTZ trial WBRT is still widely used among European radiation oncology experts.
\end{abstract}

DOI: https://doi.org/10.1016/j.radonc.2020.10.043

Posted at the Zurich Open Repository and Archive, University of Zurich ZORA URL: https://doi.org/10.5167/uzh-194303

Journal Article

Accepted Version

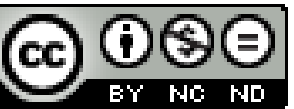

The following work is licensed under a Creative Commons: Attribution-NonCommercial-NoDerivatives 4.0 International (CC BY-NC-ND 4.0) License.

Originally published at:

Glatzer, Markus; Faivre-Finn, Corinne; De Ruysscher, Dirk; Widder, Joachim; Van Houtte, Paul; Troost, Esther G C; Slotman, Ben J; Ramella, Sara; Pöttgen, Christoph; Peeters, Stephanie T H; Nestle, Ursula; 
McDonald, Fiona; Le Pechoux, Cecile; Dziadziuszko, Rafal; Belderbos, José; Ricardi, Umberto; Manapov, Farkhad; Lievens, Yolande; Geets, Xavier; Dieckmann, Karin; Guckenberger, Matthias; Andratschke, Nicolaus; Süveg, Krisztian; Putora, Paul M (2021). Role of radiotherapy in the management of brain metastases of NSCLC - Decision criteria in clinical routine. Radiotherapy and Oncology, 154:269-273.

DOI: https://doi.org/10.1016/j.radonc.2020.10.043 


\section{Journal Pre-proofs}

Original Article

Role of radiotherapy in the management of brain metastases of NSCLC - Decision criteria in clinical routine

Markus Glatzer, Corinne Faivre-Finn, Dirk De Ruysscher, Joachim Widder, Paul Van Houtte, Esther G.C. Troost, Ben J. Slotman, Sara Ramella, Christoph Pöttgen, Stephanie T.H. Peeters, Ursula Nestle, Fiona McDonald, Cecile Le Pechoux, Rafal Dziadziuszko, José Belderbos, Umberto Ricardi, Farkhad Manapov, Yolande Lievens, Xavier Geets, Karin Dieckmann, Matthias Guckenberger, Nicolaus Andratschke, Krisztian Süveg, Paul M.

Putora

PII: S0167-8140(20)30892-6

DOI: https://doi.org/10.1016/j.radonc.2020.10.043

Reference: RADION 8611

To appear in: Radiotherapy and Oncology

Received Date: $\quad 2$ October 2020

Revised Date: $\quad 27$ October 2020

Accepted Date: $\quad 31$ October 2020

Please cite this article as: Glatzer, M., Faivre-Finn, C., De Ruysscher, D., Widder, J., Van Houtte, P., Troost, E.G.C., Slotman, B.J., Ramella, S., Pöttgen, C., Peeters, S.T.H., Nestle, U., McDonald, F., Le Pechoux, C., Dziadziuszko, R., Belderbos, J., Ricardi, U., Manapov, F., Lievens, Y., Geets, X., Dieckmann, K., Guckenberger, M., Andratschke, N., Süveg, K., Putora, P.M., Role of radiotherapy in the management of brain metastases of NSCLC - Decision criteria in clinical routine, Radiotherapy and Oncology (2020), doi: https://doi.org/10.1016/ j.radonc.2020.10.043

This is a PDF file of an article that has undergone enhancements after acceptance, such as the addition of a cover page and metadata, and formatting for readability, but it is not yet the definitive version of record. This version will undergo additional copyediting, typesetting and review before it is published in its final form, but we are providing this version to give early visibility of the article. Please note that, during the production process, errors may be discovered which could affect the content, and all legal disclaimers that apply to the journal pertain. 


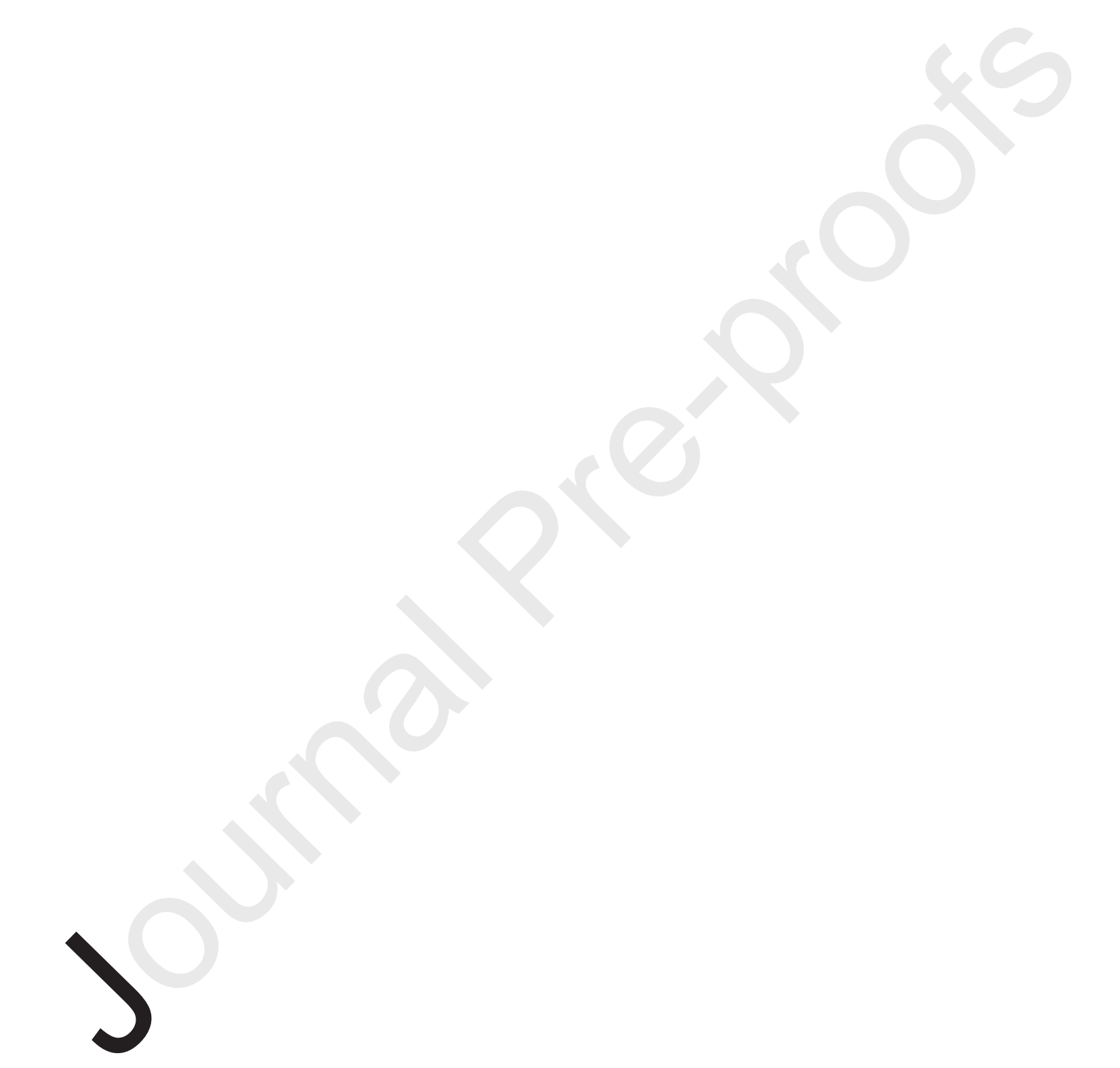




\section{Role of radiotherapy in the management of brain metastases of NSCLC - Decision criteria in}

\section{clinical routine}

Markus Glatzer ${ }^{1}$, Corinne Faivre-Finn ${ }^{2}$, Dirk De Ruysscher ${ }^{3}$, Joachim Widder ${ }^{4}$, Paul Van Houtte ${ }^{5}$, Esther G.C. Troost ${ }^{6-10}$, Ben J. Slotman ${ }^{11}$, Sara Ramella ${ }^{12}$, Christoph Pöttgen ${ }^{13}$, Stephanie T.H. Peeters ${ }^{3}$, Ursula Nestle $^{14,15}$, Fiona McDonald ${ }^{16}$, Cecile Le Pechoux ${ }^{17}$, Rafal Dziadziuszko ${ }^{18}$, José Belderbos ${ }^{19}$, Umberto Ricardi $^{20}$, Farkhad Manapov ${ }^{21}$, Yolande Lievens ${ }^{22}$, Xavier Geets ${ }^{23}$, Karin Dieckmann ${ }^{4}$, Matthias Guckenberger $^{24}$, Nicolaus Andratschke ${ }^{24}$, Krisztian Süveg ${ }^{1}$, Paul M. Putora ${ }^{1,25}$

1 Department of Radiation Oncology, Kantonsspital St. Gallen, St. Gallen, Switzerland

2 Division of Cancer Sciences, University of Manchester \& The Christie NHS Foundation Trust Manchester, Manchester, UK.

3 Maastricht University Medical Center, Department of Radiation Oncology (Maastro Clinic), School for Oncology and Developmental Biology (GROW), Maastricht, The Netherlands

4 Department of Radiation Oncology, Comprehensive Cancer Center, Medical University of Vienna, Austria

5 Department of Radiation Oncology, Institut Bordet, Université Libre Bruxelles, Belgium

6 Department of Radiotherapy and Radiation Oncology, Faculty of Medicine and University Hospital Carl Gustav Carus, Technische Universität Dresden,

Dresden, Germany.

7 Institute of Radiooncology - OncoRay Helmholtz-Zentrum Dresden - Rossendorf, Dresden, Germany.

8 OncoRay, National Center for Radiation Research in Oncology, Dresden, Germany.

9 German Cancer Consortium (DKTK), partnersite Dresden, Dresden, and German Cancer Research Center (DKFZ), Heidelberg, Germany.

10 National Center for Tumour Diseases (NCT), Partner Site Dresden, Dresden, Germany.

11 Department of Radiation Oncology, Amsterdam University Medical Center, VUMC, Amsterdam, The Netherlands

12 Department of Radiation Oncology, Campus Bio-Medico University, Rome, Italy

13 Department of Radiation Oncology, West German Tumor Centre, University of Duisburg-Essen Medical School, Germany

14 Department of Radiation Oncology, Kliniken Maria Hilf, Moenchengladbach, Germany

15 Department of Radiation Oncology, University Hospital Freiburg, Germany

16 Department of Radiotherapy, The Royal Marsden NHS Foundation Trust, London, UK

17 Departement Oncologie Radiotherapie, Gustave Roussy, Villejuif, France

18 Department of Oncology and Radiotherapy, Gdansk, Poland

19 Department of Radiation Oncology, The Netherlands Cancer Institute, Amsterdam, The Netherlands

20 Department of Oncology, University of Turin, Via Genova 3, 10126, Turin, Italy

21 Department of Radiotherapy and Radiation Oncology, University Hospital, LMU Munich, Munich, Germany

22 Radiation Oncology Department, Ghent University Hospital and Ghent University, Ghent, Belgium

23 Department of Radiation Oncology, Cliniques universitaires Saint-Luc, MIRO - IREC Lab, UCL, Belgium.

24 Department of Radiation Oncology, University Hospital Zurich, University of Zurich, Zurich, Switzerland.

25 Department of Radiation Oncology, University of Bern, Bern, Switzerland

\section{Corresponding Author:}

Dr.med.univ. Markus Glatzer

Department of Radiation Oncology, Kantonsspital St.Gallen

CH-9007 St.Gallen, Switzerland

Markus.glatzer@kssg.ch 


\begin{abstract}
Background: Whole brain radiotherapy (WBRT) is a common treatment option for brain metastases secondary to non-small cell lung cancer (NSCLC). Data from the QUARTZ trial suggest that WBRT can be omitted in selected patients and treated with optimal supportive care alone. Nevertheless, WBRT is still widely used to treat brain metastases secondary to NSCLC. We analysed decision criteria influencing the selection for WBRT among European radiation oncology experts. Methods: 22 European radiation oncologist experts in lung cancer as selected by the European Society for Therapeutic Radiation Oncology (ESTRO) for previous projects and by the Advisory Committee on Radiation Oncology Practice (ACROP) were asked to describe their strategies in the management of brain metastases of NSCLC. Treatment strategies were subsequently converted into decision trees and analysed for agreement and discrepancies. Results: 9 decision criteria (suitability for SRS, performance status, symptoms, eligibility for targeted therapy, extra-cranial tumour control, age, prognostic scores and "Zugzwang" (the compulsion to treat)) were identified. WBRT was recommended by a majority of the European experts for symptomatic patients not suitable for radiosurgery or fractionated stereotactic radiotherapy. There was also a tendency to use WBRT in the ALK/EGFR/ROS1 negative NSCLC setting. Conclusion: Despite the results of the QUARTZ trial WBRT is still widely used among European radiation oncologist experts.
\end{abstract}




\section{Introduction}

Lung cancer is the leading cause of cancer death worldwide. Non-small cell lung cancer (NSCLC) accounts for approximately $85 \%$ of all lung cancers (1). $10 \%$ of patients with NSCLC have brain metastases at diagnosis, and $25 \%-40 \%$ develop brain metastases during their disease (2). Approaches in the management of brain metastases include surgery, stereotactic radiosurgery (SRS), fractionated stereotactic radiotherapy, whole brain radiation therapy (WBRT), systemic treatments or best supportive care alone (3). The choice of treatment depends on various factors such as the number and volume of brain metastases, control of the extracranial disease, performance status or tumour biology (e.g. EGFR gene mutation) (4).

While the use of stereotactic radiosurgery in patients with limited volume of brain metastases has been well defined, its role in patients with multiple lesions is still a matter of debate. WBRT is a treatment of choice for patients with multiple brain lesions and is still the most commonly used treatment approach worldwide (5). The randomized QUARTZ trial investigated the use of dexamethasone and optimal supportive care (OSC) with or without whole brain irradiation in patients with NSCLC and brain metastases unsuitable for stereotactic radiotherapy. The primary endpoint of this non-inferiority trial was quality-adjusted life-years (QALYs). The study randomised 538 patients across UK and Australia. QALYs in the supportive care plus WBRT group were 46.4 days compared to 41.7 days in the group receiving supportive care alone. Overall survival in both groups was similar (HR 1.06, $95 \% \mathrm{Cl} 0.90-1.26, \mathrm{p}=0.81$ ) with a median survival for patients with WBRT of 9.2 weeks and 8.5 weeks for those treated with supportive care alone. Quality of life was similar between groups. Subgroup analyses showed a significant effect in favour of WBRT in the subgroup $<60$ years $(p=0.0062)$ while no significant effect of WBRT was observed in other subgroups (stratified according to gender, Karnofsky Performance Status, disease control, presence of extra-cranial metastases) (6). WBRT could therefore be omitted in most patients and optimal supportive care alone seems to be as effective. The study was performed before the era of routine molecular testing in NSCLC.

In clinical routine many criteria influence the decision-making process. The aim of this study was to identify relevant criteria in the complex process of patient selection and decision-making for the management of patients with brain metastases from NSCLC. As there are different ways of performing WBRT (different dose schedules and different techniques) prescribed dose and the use of hippocampal avoidance (HA) were also evaluated.

\section{Methods}

We asked 22 radiation oncologists who were identified by the European Society for Radiatiotherapy and Oncology (ESTRO) and by the Advisory Committee on Radiation Oncology Practice (ACROP) as experts in the field of lung cancer to participate in this analysis. Each expert was asked the following 
question: „Please describe if and for which patients with NSCLC you would recommend WBRT. Please describe any criteria used in your decision-making." As there are various dose schedules and the option to avoid the hippocampal region ( $\mathrm{HA})$ we also asked the experts to provide their answer to the following question: „Please describe your dose, technique and whether you use HA.” Answers were allowed in any format (e.g. free text, tables, diagrams or figures). No specific clinical scenarios, examples or decision criteria were proposed in order to avoid influencing responses. After the initial collection of all responses, treatment strategies were converted into decision trees, and decision criteria were extracted (7). To enable cross-comparison of algorithms, compatible criteria are a prerequisite. Similar decision criteria were fused into new comprehensive categories. For example, criteria such as fast progression of brain metastases, progression of brain metastases only under systemic treatment, high tumour load in the brain, or brain metastases near critical structures (brainstem, chiasma, optic nerves) were summarised as criteria which might lead to the compulsion to treat in a timely manner, named "Zugzwang", a German word first implemented in this setting in 2015 in a decision-making analysis for renal cell carcinoma (8). Decision criteria only mentioned by four or less experts (insular criteria) were not included into the decision trees for better overview (9). Age is one example. Age was only mentioned by three radiation oncologists as a criterion relevant for decision-making and recommended WBRT only for younger patients (age $<60-70$ years). Another factor only used by very few experts for decision-making was a prognostic score (like RPA or GPA). It was explicitly mentioned by four experts, thus this factor was also not included in the final decision trees.

Consensus and discrepancies were evaluated with the objective consensus methodology as previously described $(7,10,11)$. When 14 or more of the experts $(>60 \%)$ recommended the same we defined it as consensus.

\section{Results}

22 experts provided their decision-making and treatment strategies.

Radiation doses used for WBRT were 30Gy in 10 fractions, $20 \mathrm{~Gy}$ in 5 fractions or $12 \times 2.5 \mathrm{~Gy} / 4.25 \mathrm{~Gy}$ (simultaneous integrated boost (SIB)) as used in the phase II HIPPORAD trial (12). Most of the experts (17 out of $22,77 \%$ ) used the $10 \times 3 \mathrm{~Gy}$ fractionation regimen in daily routine. Eight radiation oncologists (36\%) always used 10x3Gy for WBRT, 6 (27\%) preferred 10x3Gy but for patients with reduced performance status or for patients who would need to start systemic treatments promptly they also offered 5x4Gy. Three centres (14\%) treated brain metastases within the HIPPORAD trial (a prospective, randomized, two-arm phase II multicentre trial comparing the impact of HA on neurocognitive failure after HA-WBRT+SIB versus WBRT+SIB in patients with multiple brain metastases) or analogous to this 
trial (12). For patients not qualifying for the HIPPORAD trial two of the experts (9\%) used WBRT with 10x3Gy instead, and one radiation oncologist (4\%) offered 10x3Gy or 5x4Gy depending on performance status. 5 out of our 22 experts always recommended 5x4Gy for WBRT.

The preferred technique for WBRT was a standard 3D technique with opposing fields $+/$ - field in field technique. HA-WBRT or WBRT with SIB was performed either using helical IMRT with Tomotherapy or VMAT. Seven experts (32\%) offered HA in clinical routine outside of trials. Among these experts, 2 always performed HA-WBRT when WBRT was performed, 3 experts recommended HA only for very fit patients with a relatively long life expectancy with good systemic treatment options and a limited number of brain metastases, and 2 radiation oncologists used HA-WBRT only in the re-irradiation setting for progressive disease in the brain after stereotactic radiotherapy. A contraindication for HAWBRT in all centres offering it, was the location of brain metastases near the hippocampus.

The decision criteria used for the management of brain metastases in NSCLC included: suitability for SRS, performance status, symptoms, eligibility for targeted therapy (ALK+, EGFR+ or ROS1+ tumour profiles or PDL1 expression), extra-cranial tumour control (present or achievable), age, prognostic scores and "Zugzwang" (the compulsion to treat (8)) (Table 1). Suitability for SRS was defined very heterogeneously among experts. One expert performed SRS in patients with up to 15 brain metastases, 4 experts recommended SRS for up to 10 metastases, for 3 experts the cut-off was 5 metastases, 6 experts recommended SRS in patients with 4 or less metastases and 3 experts only performed SRS in case of 3 or less metastases. In summary, more than $70 \%$ of the experts who used the number of brain metastases for decision-making, would do SRS only in patients with up to 5 brain metastases (Figure 1). While the majority of experts (77\%) used the number of brain metastases for decision-making, for 5 experts the cumulative volume of brain metastases (e.g. $<20 \mathrm{~cm} 3$ ) was more crucial. However, in case of a limited number or volume of brain metastases all experts recommended a local treatment, either SRS, fractionated stereotactic radiotherapy or surgery. A worse performance status, defined as Karnofsky Performance Status (KPS) $<70$ by the majority of our experts, was a contraindication for radiotherapy to the brain. One expert defined "worse" performance status as KPS of $\leq 50$.

\begin{tabular}{|c|c|c|c|c|c|c|c|c|c|c|c|}
\hline & \multirow[t]{2}{*}{ age } & \multirow{2}{*}{$\begin{array}{l}\text { volume/number } \\
\text { of brain } \\
\text { metastases }\end{array}$} & \multirow{2}{*}{$\begin{array}{l}\text { prognostic } \\
\text { scores }\end{array}$} & \multirow{2}{*}{$\begin{array}{l}\text { extracranial } \\
\text { control }\end{array}$} & \multirow[t]{2}{*}{ PS } & \multirow{2}{*}{$\begin{array}{l}\text { eligibility for } \\
\text { targeted } \\
\text { therapy }\end{array}$} & \multirow[t]{2}{*}{ symptoms } & \multicolumn{4}{|c|}{ «Zugzwang» } \\
\hline & & & & & & & & $\begin{array}{l}\text { critical } \\
\text { location }\end{array}$ & $\begin{array}{l}\text { critical } \\
\text { size }\end{array}$ & $\begin{array}{l}\text { fast } \\
\text { progression }\end{array}$ & $\begin{array}{l}\text { pressure } \\
\text { of the } \\
\text { referring } \\
\text { physicians }\end{array}$ \\
\hline A & $x$ & $x$ & $x$ & $x$ & $x$ & $x$ & & $x$ & & & \\
\hline B & & $x$ & & & $x$ & & $x$ & $x$ & & & $x$ \\
\hline C & & $x$ & & & $x$ & $x$ & $x$ & & & & $x$ \\
\hline D & & $x$ & & $x$ & $x$ & & & & & & \\
\hline $\mathbf{E}$ & & $x$ & & & $x$ & $x$ & $x$ & & & $x$ & \\
\hline $\mathbf{F}$ & $x$ & $x$ & & $x$ & $x$ & $x$ & $x$ & & & & \\
\hline G & & $x$ & $x$ & & $x$ & $x$ & & & & & \\
\hline $\mathbf{H}$ & & $x$ & $x$ & $x$ & $x$ & $x$ & & & & & \\
\hline I & & $x$ & & & $x$ & & & & & & \\
\hline
\end{tabular}




\begin{tabular}{|c|c|c|c|c|c|c|c|c|c|c|}
\hline $\mathbf{J}$ & & $x$ & & & $x$ & & $x$ & & & \\
\hline K & & $x$ & & $x$ & $x$ & & $x$ & & & \\
\hline $\mathbf{L}$ & & $x$ & & & $x$ & $x$ & $x$ & & & \\
\hline $\mathbf{M}$ & & $x$ & & & $x$ & & & & & \\
\hline $\mathbf{N}$ & $x$ & $x$ & & & $x$ & & $x$ & & & \\
\hline 0 & & $x$ & & $x$ & $x$ & & & & & $x$ \\
\hline $\mathbf{P}$ & & $x$ & & & $x$ & $x$ & $x$ & & & \\
\hline $\mathbf{Q}$ & & $x$ & $x$ & $x$ & $x$ & & $x$ & & & $x$ \\
\hline $\mathbf{R}$ & & $x$ & & & $x$ & & & & & \\
\hline $\mathbf{S}$ & & $x$ & & & $x$ & & $x$ & $x$ & $x$ & $x$ \\
\hline $\mathbf{T}$ & & $x$ & & & $x$ & $x$ & & $x$ & $x$ & \\
\hline $\mathbf{U}$ & & $x$ & & & $x$ & & & & & 8 \\
\hline $\mathbf{V}$ & & $x$ & & & $x$ & & & & & \\
\hline
\end{tabular}

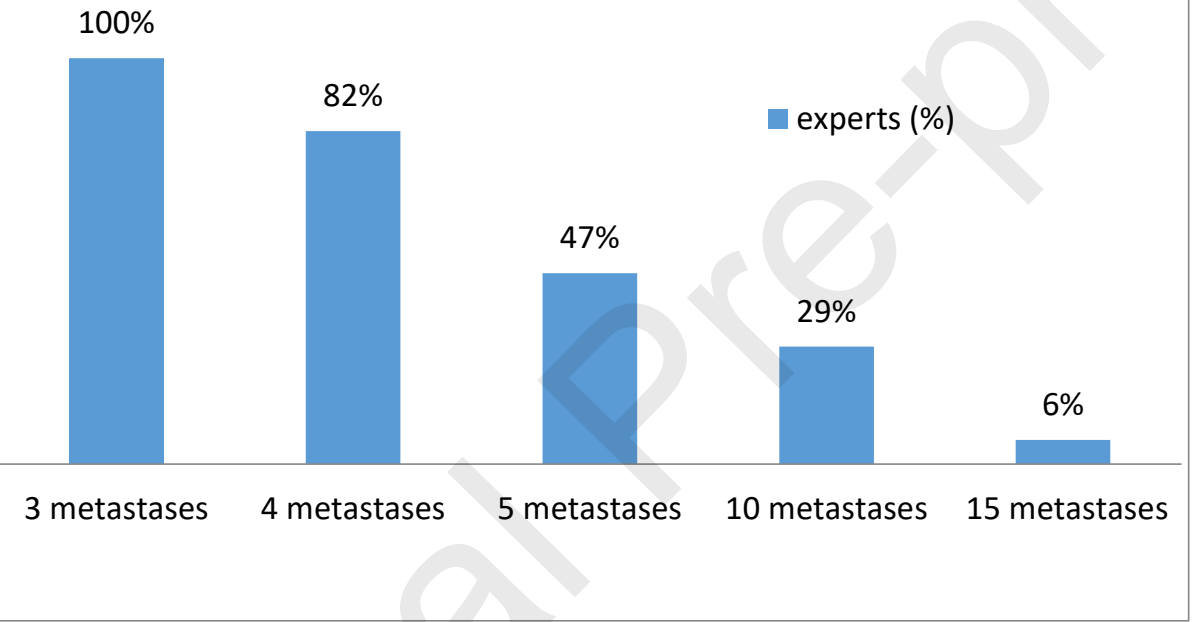

Figure 1. Definition of "suitability for SRS" by the experts. The more brain metastases the less experts recommended stereotactic radiosurgery. In up to 3 metastases, all experts considered SRS, for one expert (6\%) SRS was recommended for up to 15 brain metastases.

The majority of participating radiation oncologists recommended WBRT for symptomatic brain metastases when targeted therapies like TKI or immuno-oncology agents were not an option, regardless of extra-cranial tumour control (Figure 2). Two experts explicitly mentioned that they would perform WBRT only after improvement of symptoms after initiation of corticosteroids. In patients who are candidates for targeted therapies (ALK+, EGFR+, ROS1+ or PDL1 expression) 64\% of experts would prefer starting with targeted therapy, e.g. TKI alone, and only when metastases in the brain progress ("Zugzwang") they would recommend WBRT. For asymptomatic patients the trend for WBRT decreases. Most of the experts avoid WBRT in asymptomatic patients with driver mutation positive tumours, except if the tumour progresses in the brain under systemic therapy. For $32 \%$ of our experts the location of brain metastases near critical structures in the brain, very large metastases or a fast 
progression of brain metastases (summarized as Zugzwang) was an indication for WBRT even if effective systemic treatment options were available.

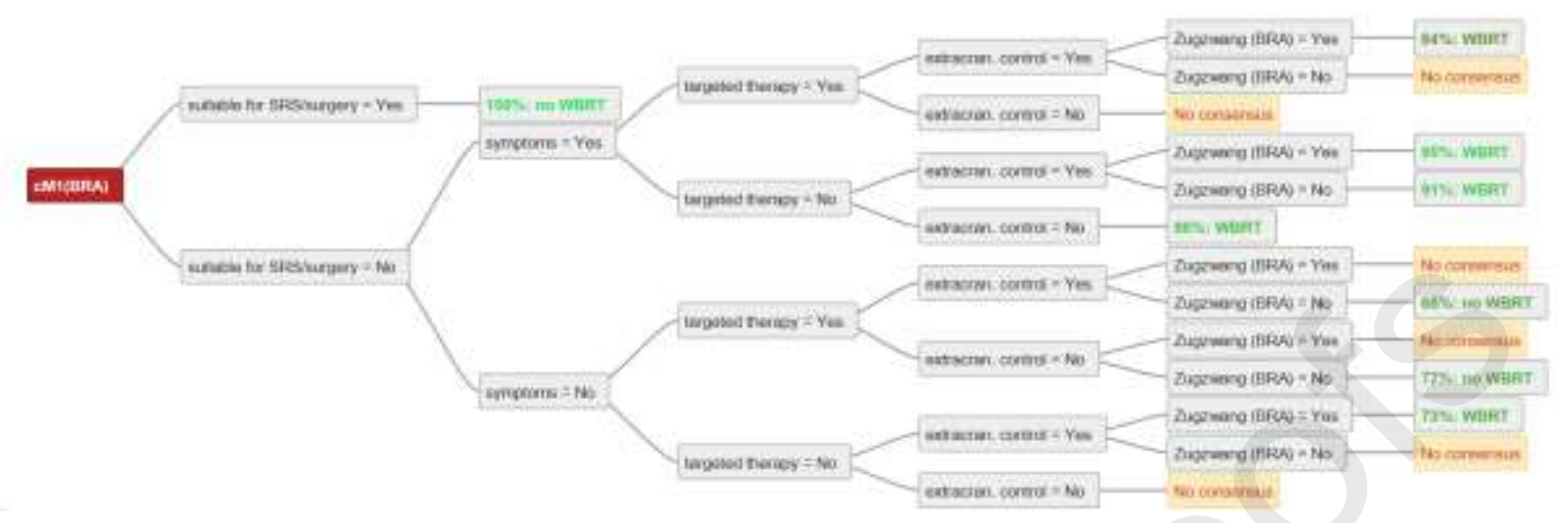

Figure 2. Consensus for WBRT/noWBRT. No consensus $=13$ or less experts ( $<60 \%)$. BRA - Brain, Zugzwang - the compulsion to treat.

\section{Discussion}

For a limited number and/or volume of brain metastases a focal therapy (surgery, radiosurgery, and fractionated radiotherapy) seems to be the treatment of choice as highlighted by our analysis. All of our experts recommend a local treatment for limited brain metastases. The definition of "limited" varies among the experts, and in literature, where no universally accepted definition exists (13). For most of our experts "limited" was defined as 4 or less brain metastases. When metastases cannot reasonably be treated with local treatments, WBRT is a treatment option. This decision-making analysis shows that WBRT is still widely used, especially for symptomatic patients. However, the use of WBRT in the management of brain metastases of NSCLC decreases. This is mainly due to more effective drugs, e.g. targeted therapies, but also due to improved radiation techniques allowing for a better sparing of macroscopically unaffected brain tissue. The data of the QUARTZ trial for patients that were not candidates for SRS, demonstrating no significant QALY benefit for WBRT compared to OSC alone, must also play a role in the decision making process (6). Some subgroups of patients, e.g. patients with good KPS $(\geq 70 \%)$ and age $<60$, in general patients with good prognostic scores (e.g. RPA or GPA score) derived more clinical benefit from WBRT. Age was only used by 3 of our experts and the use of a score (GPA and/or RPA) was explicitly mentioned by 4 experts as decision-making criterion. All experts included KPS/PS in their decision-making analysis and a poor performance status was a contraindication for WBRT for all of our experts. The main selection criterion for patients in the QUARTZ trial was the uncertainty in the clinicians' or patients' minds about the potential benefit of WBRT. This resulted in a very unfavourable selection of NSCLC patients with a high proportion of patients with uncontrolled primary and extracranial metastases, overall, patients with a poor prognosis. Thus, it seems these criteria are closely considered in clinical routine. In our analyses, more than half of the experts did so. Most of the experts in our analysis used extra-cranial disease control 
and also the eligibility for targeted therapy for their decision-making. Especially for controlled extracranial disease most of our experts recommended WBRT in patients unsuitable for SRS, as patients with favourable prognosis benefit from WBRT. This is in-line with published literature:Agarwal et al (14) investigated prognostic factors to identify patients with poor prognosis who may not benefit from WBRT and translated the factors into a prognostic model. They mentioned EGFR mutation, KPS and gender as significant prognostic factors of overall survival. Patients with poor performance status, male gender or EGFR wildtype NSCLC had a median overall survival of 67 days and therefore may not benefit from WBRT.

Another limitation of the QUARTZ trial is that most patients did not receive systemic therapy. As many targeted therapies (e.g. for EGFR-mutant or ALK positive lung cancers) have a good CNS penetration with more than $70 \%$ intracranial response rate (15), there is a trend for omission of WBRT, especially in patients with NSCLC and suitable for targeted agents. This was highlighted in this analysis as some of our experts only recommend WBRT in patients who are not eligible for targeted therapy. In the past decade new targeted therapies and immunotherapies for patients with NSCLC changed the treatment approaches and outcome of patients with brain metastases considerably.

Molecular characteristics and KPS/PS were also identified in our analysis as factors used by our experts for their decision-making process.

Interestingly, some of our experts also used tumor growth or the location of the brain metastases for decision-making. While the static situation of the tumor might fulfill the criterion to rather defensive or passive approach, clinicians identify factors such as fast growth or critical location as a compulsion to treat actively (Zugzwang). Next to commonly used decision-criteria (as used in clinical trials and databases), the concept of Zugzwang allowed for a representation of a certain urgency which is commonly interpreted by the clinician.

The variability of decision criteria among the participating experts may have multiple causes. These may include local traditions, level of individual experience as well as different guidelines used for development of local recommendation of the interdisciplinary tumour board. The aim of this study was to assess criteria in the complex process of patient selection for the management of brain metastases in NSCLC patients. This survey is only a surrogate and should give an impression on which criteria are used and which treatment decision are made. The clinical decision-making process is rarely a 1:1 extraction from published data, this is why we believe this work adds to the understanding of how literature is applied to clinical practice.

The prescribed radiation doses for WBRT in this analysis included 10x3Gy, 5x4Gy or dose regimens with SIB. Current guidelines $(16,17)$ recommend WBRT dose schemes of 20 - 40Gy in 5-20 fractions. 
The most frequent WBRT schedules are 20Gy in 5 fractions or 30 Gy in 10 fractions, with no difference in outcome (18). The most common fractionation regime used by experts in our study was $30 \mathrm{~Gy}$ in 10 fractions or $20 \mathrm{~Gy}$ in 5 fractions, for patients with reduced performance status. Very few experts treated brain metastases within or accordingly to the ongoing randomized phase II HIPPORAD trial (12). The aim of this trial is to assess the efficacy and safety of WBRT with HA combined with a SIB to metastases/resection cavities. Much of the concern over WBRT relates to associated toxicities which include cognitive deficits (19). Radiation dose to the neuro-regenerative zone of the hippocampus seems to be associated with cognitive decline. Hippocampal avoidance is hypothesized to preserve cognition. The recently published randomized study by Brown et al $(20)$ showed better preservation of cognitive function and patient-reported symptoms, with no difference in intracranial PFS and OS, when using HA-WBRT and concurrent memantine compared to WBRT and memantine in patients with brain metastases. As only in $2 \%$ of patients metastases will appear in the hippocampus and up to $8 \%$ in the perihippocampal area, the approach of HA should be considered as a treatement option (21). However, as there are other data showing no significant difference in neurocognition between HA WBRT compared to standard WBRT (22), HA is not universally accepted. This is also conveyed in this analysis as two thirds of experts to do not use HA-WBRT in clinical routine. However, there are various trials in progress.

In summary, WBRT is still widely used among European thoracic radiation oncology experts with a trend towards the use of WBRT for symptomatic brain metastases who cannot be treated with stereotactic radiotherapy. 10x3Gy was the most commonly used fractionation regimen among the experts. For patients with asymptomatic brain metastases and suitable for targeted therapies, the majority of our lung cancer experts recommended a watchful waiting strategy with regards to radiotherapy treatment. 


\section{Declaration of Competing Interest}

The authors declare that they have no known competing financial interests or personal relationships that could have appeared to influence the work reported in this paper.

\section{Acknowledgment}

Prof Corinne Faivre-Finn was supported by a grant from the NIHR Manchester Biomedical Research Centre.

\section{Conflicts of interest}

PM Putora - research support and educational grants to the department: AstraZeneca, Celgene, Takeda.

F. McDonald - consulting fees and speaker fees by AstraZeneca, speaker fees by Elekta and consulting fees by Accuray. B.J. Slotman - research funding from Varian medical systems and ViewRay Inc.,

C Le Pechoux: Grants to the institution from Astra-Zeneca, Nanobiotix, Roche (for Advisory Boards) and Amgen, Eli-Lilly, Medscape (for educational meetings), PriMEOncology (Speaker fees).

S. Ramella: Advisory Boards Astra Zeneca, Ipsen and Roche. Dirk De Ruysscher: Advisory role (all institution payments): Bristol-Myers Squibb, Celgene, Merck/Pfizer, Roche/Genentech, AstraZeneca, MSD, Seattle genetics. Research funding (all institution payments): Boehringer Ingelheim, BristolMyers Squibb, AstraZeneca, Philips, Olink.

U. Ricardi: consulting fees and speaker fees by AstraZeneca

R Dziadziuszko - consulting fees for advisory boards or lectures from Roche, AstraZeneca, Pfizer, Novartis, Boehriner-Ingelheim, Takeda, FoundationMedicine, MSD and SeattleGenetics.

C Faivre-Finn: consulting fees, speaker fees and research funding by AstraZeneca, research funding by Elekta

Y. Lievens: consulting and speakers fees by AstraZeneca and RaySearch But there are no conflicts of interest to declare related to the topic of the manuscript. 
References

1. Herbst RS, Morgensztern D, Boshoff $C$. The biology and management of non-small cell lung cancer. Nature. 2018;553(7689):446-54.

2. Barnholtz-Sloan JS, Sloan AE, Davis FG, Vigneau FD, Lai P, Sawaya RE. Incidence proportions of brain metastases in patients diagnosed (1973 to 2001) in the Metropolitan Detroit Cancer Surveillance System. J Clin Oncol. 2004;22(14):2865-72.

3. Liu Q, Tong, X., Wang, J. . Management of brain metastases: history and the present. Chin Neurosurg J. 2019;5:1.

4. Ernani V, Stinchcombe TE. Management of Brain Metastases in Non-Small-Cell Lung Cancer. J Oncol Pract. 2019;15(11):563-70.

5. Kraft J, Zindler J, Minniti G, Guckenberger M, Andratschke N. Stereotactic Radiosurgery for Multiple Brain Metastases. Curr Treat Options Neurol. 2019;21(2):6.

6. Mulvenna P, Nankivell M, Barton R, Faivre-Finn C, Wilson P, McColl E, et al. Dexamethasone and supportive care with or without whole brain radiotherapy in treating patients with non-small cell lung cancer with brain metastases unsuitable for resection or stereotactic radiotherapy (QUARTZ): results from a phase 3, non-inferiority, randomised trial. Lancet. 2016;388(10055):2004-14.

7. Panje CM, Glatzer M, von Rappard J, Rothermundt C, Hundsberger T, Zumstein V, et al. Applied Swarm-based medicine: collecting decision trees for patterns of algorithms analysis. BMC Med Res Methodol. 2017;17(1):123.

8. Rothermundt C, Bailey A, Cerbone L, Eisen T, Escudier B, Gillessen S, et al. Algorithms in the First-Line Treatment of Metastatic Clear Cell Renal Cell Carcinoma--Analysis Using Diagnostic Nodes. Oncologist. 2015;20(9):1028-35.

9. Iseli T, Fischer GF, Panje CM, Glatzer M, Hundsberger T, Rothermundt C, et al. Insular Decision Criteria in Clinical Practice: Analysis of Decision-Making in Oncology. Oncology. 2020;98(6):438-44.

10. Putora PM, Panje CM, Papachristofilou A, Dal Pra A, Hundsberger T, Plasswilm L. Objective consensus from decision trees. Radiat Oncol. 2014;9:270.

11. Glatzer M, Faivre-Finn C, De Ruysscher D, Widder J, Van Houtte P, Troost EGC, et al. Once daily versus twice-daily radiotherapy in the management of limited disease small cell lung cancer Decision criteria in routine practise. Radiother Oncol. 2020;150:26-9.

12. Grosu AL, Frings L, Bentsalo I, Oehlke O, Brenner F, Bilger A, et al. Whole-brain irradiation with hippocampal sparing and dose escalation on metastases: neurocognitive testing and biological imaging (HIPPORAD) - a phase II prospective randomized multicenter trial (NOA-14, ARO 2015-3, DKTK-ROG). BMC Cancer. 2020;20(1):532.

13. Izard MA, Moutrie V, Rogers JM, Beath K, Grace M, Karle B, et al. Volume not number of metastases: Gamma Knife radiosurgery management of intracranial lesions from an Australian perspective. Radiother Oncol. 2019;133:43-9.

14. Agarwal JP, Chakraborty S, Laskar SG, Mummudi N, Patil VM, Upasani M, et al. Applying the QUARTZ Trial Results in Clinical Practice: Development of a Prognostic Model Predicting Poor Outcomes for Non-small Cell Lung Cancers with Brain Metastases. Clin Oncol (R Coll Radiol). 2018;30(6):382-90.

15. Wang C, Lu X, Lyu Z, Bi N, Wang L. Comparison of up-front radiotherapy and TKI with TKI alone for NSCLC with brain metastases and EGFR mutation: A meta-analysis. Lung Cancer. 2018;122:94-9.

16. Network. NCC. NCCN Clinical Practice Guidelines in Oncology: Central Nervous System Cancers, Version.2.2018. Available at http://www.nccn.org/professionals/physician gls/PDF/cns.pdf. 2018.

17. Planchard D, Popat S, Kerr K, Novello S, Smit EF, Faivre-Finn C, et al. Metastatic non-small cell lung cancer: ESMO Clinical Practice Guidelines for diagnosis, treatment and follow-up. Ann Oncol. 2018;29 Suppl 4:iv192-iv237. 
18. Tsao MN, Xu W, Wong RK, Lloyd N, Laperriere N, Sahgal A, et al. Whole brain radiotherapy for the treatment of newly diagnosed multiple brain metastases. Cochrane Database Syst Rev. 2018;1:CD003869.

19. Brown PD, Ballman KV, Cerhan JH, Anderson SK, Carrero XW, Whitton AC, et al. Postoperative stereotactic radiosurgery compared with whole brain radiotherapy for resected metastatic brain disease (NCCTG N107C/CEC.3): a multicentre, randomised, controlled, phase 3 trial. Lancet Oncol. 2017;18(8):1049-60.

20. Brown PD, Gondi V, Pugh S, Tome WA, Wefel JS, Armstrong TS, et al. Hippocampal Avoidance During Whole-Brain Radiotherapy Plus Memantine for Patients With Brain Metastases: Phase III Trial NRG Oncology CC001. J Clin Oncol. 2020;38(10):1019-29.

21. Gondi V, Tome WA, Mehta MP. Why avoid the hippocampus? A comprehensive review. Radiother Oncol. 2010;97(3):370-6.

22. Mayinger $\mathrm{M}$, Andratschke N. [Role of hippocampal avoidance during therapeutic whole-brain radiotherapy]. Strahlenther Onkol. 2020. 


\section{Highlights}

1. WBRT is still widely used among European radiation oncology experts with a trend towards the use of WBRT for symptomatic brain metastases and contraindication for stereotactic radiotherapy.

2. For patients with asymptomatic brain metastases and option for targeted therapies, a watchful waiting strategy with regards to radiotherapy treatment is a common strategy among European experts.

3. WBRT with 10x3Gy was the most commonly used fractionation regimen among the experts. 